Research Paper

\title{
Immunohistochemical study of hair follicle stem cells in regenerated hair follicles induced by Wnt $10 \mathrm{~b}$
}

\author{
Yiming Zhang1, 2, Yizhan Xing2, Haiying Guo², Xiaogen $\mathrm{Ma}^{2}$, Yuhong Li ${ }^{2}$ \\ 1. Department of Plastic and Cosmetic surgery, Xinqiao Hospital, Third Military Medical University, Chongqing 400037, China. \\ 2. Department of Cell Biology, Third Military Medical University, Chongqing 400038, China. \\ $\triangle$ Corresponding author: Yuhong Li, E-mail: liyuhongtmmu@hotmail.com. Tel: +862368752253.
}

(1) Ivyspring International Publisher. Reproduction is permitted for personal, noncommercial use, provided that the article is in whole, unmodified, and properly cited. See http://ivyspring.com/terms for terms and conditions.

Received: 2016.05.09; Accepted: 2016.08.22; Published: 2016.09.20

\begin{abstract}
The regulation of the periodic regeneration of hair follicles is complicated. Although Wnt10b has been reported to induce hair follicle regeneration, the characteristics of induced hair follicles, especially the target cells of Wnt 10b, have not yet been clearly elucidated. Thus, we systematically evaluated the expression and proliferation patterns of Wntlob-induced hair follicles. We found that Wnt 10b promoted the proliferation of hair follicle stem cells from 24 hours after AdWnt 10b injection. Seventy-two hours after AdWntlob injection, cells outside of bulge area began to proliferate. When the induced hair follicle entered full anagen, although the hair follicle stem cells were normal, canonical Wnt signaling was maintained in the hair precortex cells. Our results reveal that the target cells that overexpressed Wntlob included hair follicle stem cells, hair precortex cells, and matrix cells.
\end{abstract}

Key words: Wnt10b, hair follicle stem cells, cell proliferation, Wnt signaling pathway.

\section{Introduction}

As an accessory mini-organ of the skin, hair follicles begin to form during the embryonic phase and complete morphogenesis postnatally. Hair follicles undergo periodic growth over their lifetime. The hair cycle consists of anagen, catagen and telogen [1]. Hair follicle stem cells (HFSCs) are located in the bulge area just under the openings of sebaceous glands. Under normal conditions, bulge HFSCs maintain the epidermal origin for hair regeneration [2]. HFSCs in the bulge appear to be dispensable for hair regeneration after injury [3]. The periodic activation and quiescence of HFSCs is precisely regulated; several signaling pathways are involved in this process, including the wingless-type mouse mammary tumor virus integration site (Wnt) [4], bone morphogenetic protein [5], and fibroblast growth factor [6] pathways.

During telogen, the balance between activators and inhibitors may determine whether a hair follicle can reenter anagen [7], and some activators and inhibitors have been reported $[8,9]$. Previously, we reported that Wnt10b is a strong activator of the hair cycle [10]. Wnt10b can promote hair follicle growth via the canonical Wnt signaling pathway [11], and the overexpression of Wnt10b can induce hair follicle regeneration in vivo. Wnt10b has also been demonstrated to play important roles in dermal papilla cells and melanocyte stem cells $[12,13]$. Our previous study raised the following question: what is the target cell of overexpressed Wnt10b? In the present study, we found that hair follicle stem cells proliferate following Wnt10b injection and therefore conclude that these cells are among the activated targets of ectopic Wnt10b.

\section{Materials and Methods}

\section{Animals}

C57BL/ 6 mice were obtained from and housed in the laboratory animal center of the Third Military Medical University, Chongqing, China. All the animal-related procedures were conducted in strict accordance with the approved institutional animal 
care and maintenance protocols. All experimental protocols were approved by the Research Committee of the Third Military Medical University.

\section{Intradermal administration of adenoviruses}

Female mice at postnatal day 56 (telogen) were anesthetized with $1 \%$ pentobarbital sodium. Their back hairs were depilated, and $50 \mu \mathrm{L}$ of AdWnt10b ( Wnt10b mediated by an adenovirus) or AdGFP (GFP mediated by an adenovirus) was injected intradermally along the median dorsal line of the skin, at a titer of $10^{8} \mathrm{PFU} / \mathrm{ml}$. The dorsal skins of the injected areas were harvested for immunostaining at the indicated time points post-administration. At each time point, 3 mice were used for each group. The total number of mice used was 60 .

\section{Tracing proliferating cells}

BrdU (5-bromo-2-deoxyuridine, Sigma, USA, B5002) was prepared at $10 \mathrm{mg} / \mathrm{mL}$ in $0.9 \%$ sodium chloride. Four hours before sacrifice, BrdU was injected intraperitoneally into mice that had been treated with AdWnt10b or AdGFP. Samples of dorsal skins were harvested for immunostaining at the designated post-adenovirus administration time points.

\section{Immunostaining}

Immunostaining was performed on $5-\mu \mathrm{m}$ paraffin-embedded sections from the dorsal skin. The sections were deparaffinized, rehydrated, and boiled in citrate buffer solution. After blocking in $10 \%$ goat serum (in PBS), the sections were incubated with the following primary antibodies: mouse monoclonal anti- $\beta$-catenin (1:100, Santa Cruz, USA, sc-7963), rabbit polyclonal anti-Wnt10b (1:100, Santa Cruz, USA, sc-25524), mouse monoclonal anti-BrdU (1:200, Abcam, USA, ab8039), rabbit polyclonal anti-Sox4 (1:100, Santa Cruz, USA, sc-20090), mouse monoclonal anti- $\beta 1$-integrin (1:100, Santa Cruz, USA, sc-9970), and rabbit polyclonal anti-CD34 (1:100, Santa Cruz, USA, sc-9095). CY3-labeled secondary antibodies (goat anti-rabbit, A0516 and goat anti-mouse, A0521, Beyotime, China) were used for immunofluorescence, and DAPI (Beyotime, C1002) was used to counterstain the cell nuclei. An HRP-labeled goat anti-mouse detection kit (Zhongshan Goldenbridge, China, PV-6002) was used for immunohistochemistry.

\section{Statistical analysis}

The number of BrdU-positive cells was counted using Image Pro Plus 6.0. For each group, 3 mice were used, and the cells were counted in 10 representative hair follicles in different visual fields in 1 section per mouse. The representative hair follicles were defined as including the entire structure of a hair follicle, from distal hair stem to proximal dermal papilla. T-tests were used to evaluate the differences between groups.

\section{Results}

\section{Cell proliferation analysis}

To identify the target cells of overexpressed Wnt10b, we traced proliferating cells via BrdU labeling. Immunohistochemistry revealed that cell proliferation began at 24 hours after AdWnt10b injection. Proliferating cells were observed both inside and outside the bulge area of the hair follicle (Fig. 1A). From 24 hours to 52 hours post-injection, the number of proliferating cells in the bulge of hair follicles increased (Fig. 1B-1C).

To demonstrate that cells indeed express the viral product, we detected the expression of GFP at 48 hours after AdGFP injection. GFP was expressed in almost all of the cells around the injection area, including hair follicles (Fig. 2A-2B). Double staining of CD34 and BrdU revealed that some of the CD34-positive cells in the hair follicle were proliferating at 48 hours after AdWnt10b injection (Figure 2C-2D). Immunofluorescence staining with a nuclear counterstain revealed that the hair follicles were still in telogen both at 24 hours and at 48 hours after injection. Immunofluorescence staining also revealed that proliferating cells were located in the bulge area (Fig. 3).

\section{Expression patterns of HFSC markers in Wnt 10b-induced hair follicles}

To determine whether Wnt10b induces activation of HFSCs in the Wnt10b-induced anagen hair follicles, we evaluated the expression patterns of several HFSC markers. CD34 was expressed in the cytoplasm of the cells in the bulge area (Fig. 4A), and Sox4 was expressed in the lower part of the bulge area and the outer root sheath in the cytoplasm (Fig. 4B). $\beta 1$-Integrin was expressed in the cell membranes in the bulge area, and this expression extended to the outer root sheath (Fig. 4C). The expression patterns of CD34 and 31 -integrin in Wnt10b-induced anagen hair follicles were similar to those in untreated anagen hair follicles (control group), but the expression level of Sox4 was lower in the Wnt10b-induced anagen hair follicles than in the control group (Fig. 4B).

\section{Analysis of canonical Wnt signaling in the Wnt 1 Ob-induced full hair follicles}

We next evaluated the expression of Wnt $10 \mathrm{~b}$ and $\beta$-catenin via immunofluorescence in the Wnt10b-induced anagen hair follicles and compared them with normal hair follicles in the corresponding stage. The expression patterns of Wnt $10 \mathrm{~b}$ in the Wnt10b-induced hair follicles and in the control hair 
follicles were similar (Fig. 5A, 5B), whereas those of $\beta$-catenin were different (Fig. 5C, 5D). In the Wnt10b-induced hair follicles, translocation of $\beta$-catenin was observed in both the hair matrix cells and the hair precortex cells (Fig. 5C-5E). In the normal hair follicles of the corresponding stage, the translocation of $\beta$-catenin was also observed in the hair matrix cells but was markedly reduced in the cortex region.

\section{Discussion}

Wnt10b can induce hair follicle regeneration, and the duration of Wnt10b overexpression is a key factor in this process. Prolonged overexpression of
Wnt10b induces epidermal keratinocyte transformation in addition to inducing abnormal hair follicle regeneration [14], whereas transient overexpression of Wnt10b induces only hair follicle regeneration [10]. Upon prolonged overexpression, the canonical Wnt signaling pathway is constitutively activated in many cell types in the skin. However, the characteristics of hair follicles induced by transient Wnt10b overexpression are not well described, and the target cells of the Wnt10b overexpression remain to be identified. Here, we evaluated the expression patterns and proliferation characteristics of Wnt10b-induced hair follicles.
A
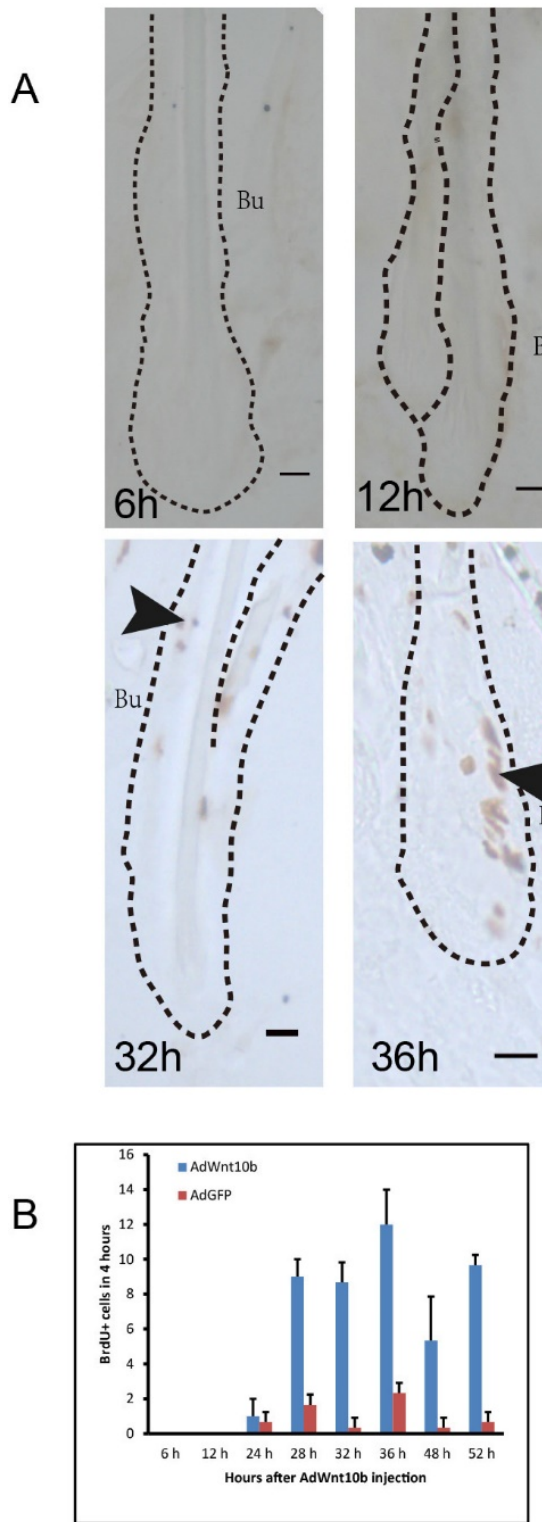
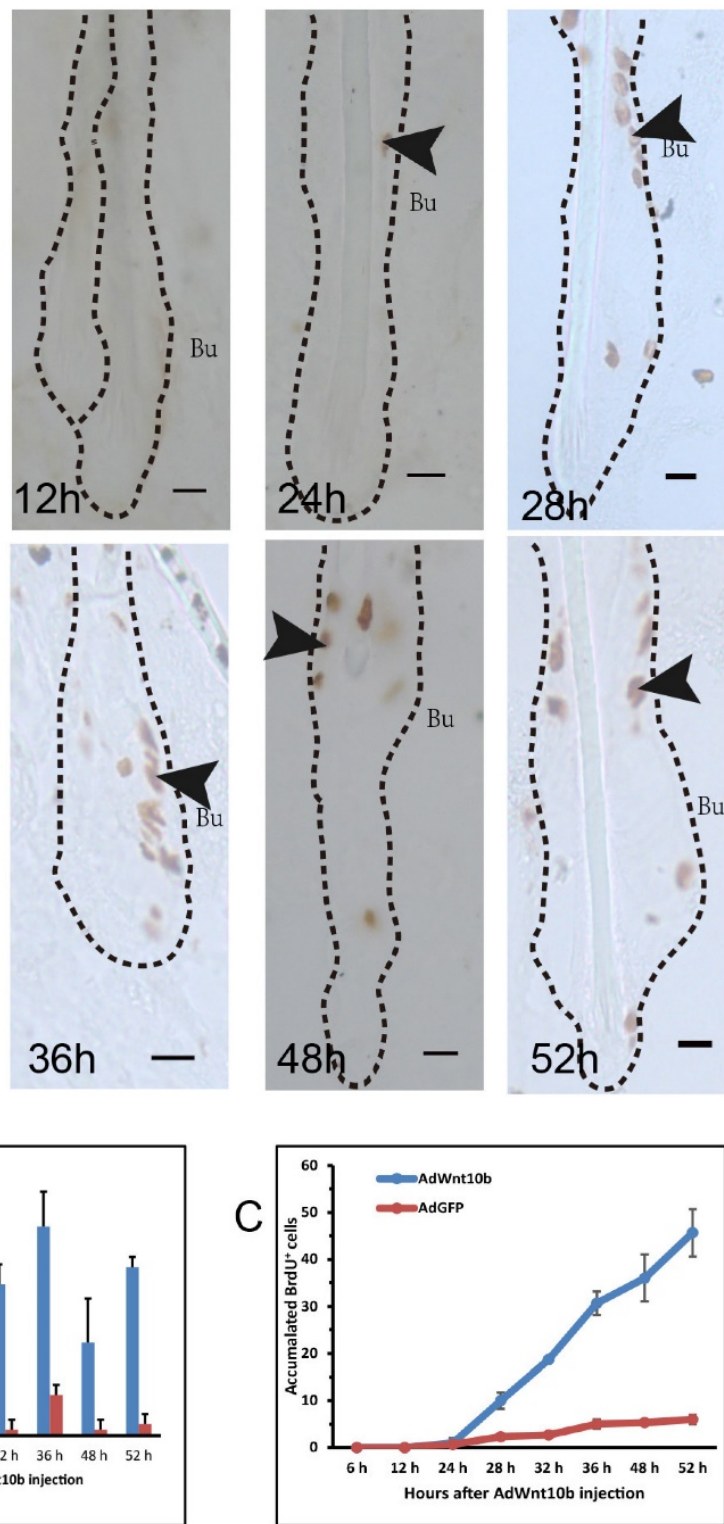

Figure 1. Immunohistochemical detection of BrdU incorporation in Wnt10b-overexpressing hair follicles. Eight-week-old C57BL/6 mice were intradermally injected with $\mathrm{AdWnt} 10 \mathrm{~b}$. BrdU was administered 4 hours before the skin was harvested. (A) BrdU expression was evaluated using immunohistochemistry at $6,12,24,28,32,36,48$, and 52 hours after injection. The arrowheads indicate the expression of BrdU. The dashed line delineates the hair follicle structure. Bu, bulge. Scale bar $=10 \mu \mathrm{m}$. $(\mathrm{B}) \mathrm{Statistics}$ regarding the numbers of BrdU+ cells at 4 hours at these time points. (C) Statistics regarding the numbers of accumulated BrdU+ cells at these time points. $\mathrm{N}=10$. 
A

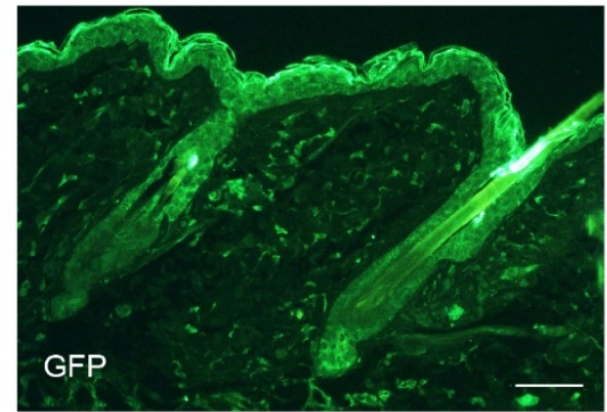

B

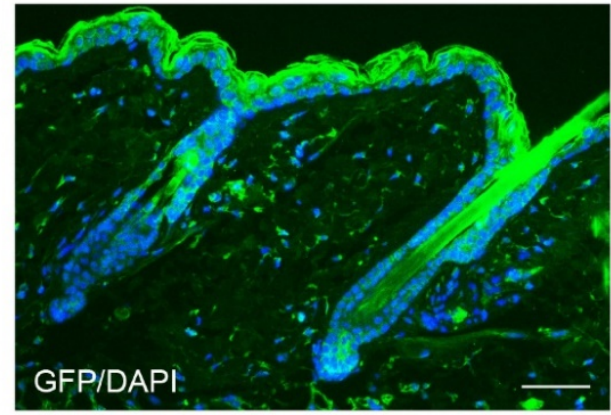

C
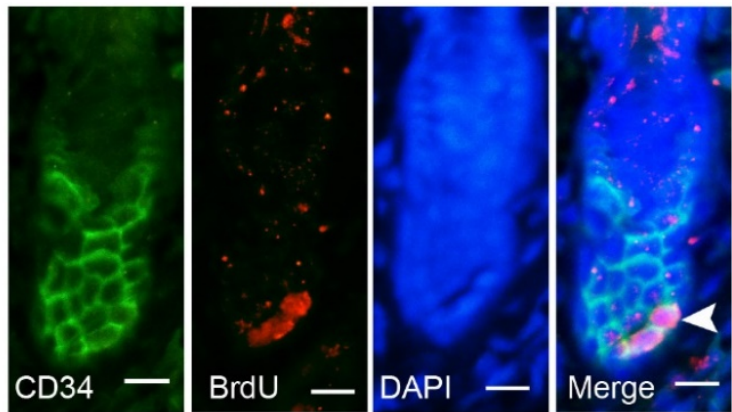

D

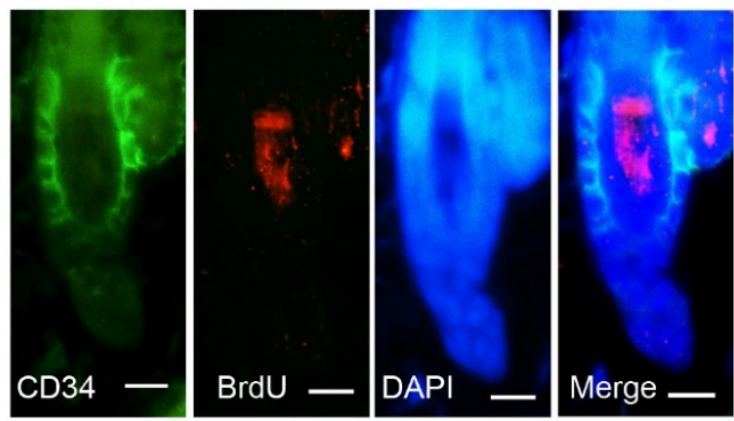

Figure 2. Immunofluorescent detection of the expression of GFP and double staining for BrdU and CD34. Eight-week-old C57BL/6 mice were intradermally injected with $\operatorname{AdGFP}(A, B, D)$ or AdWnt10b (C). (A, B) GFP expression was detected by immunofluorescence at 48 hours after injection. (C, D) BrdU was administered 4 hours before the skin was harvested. BrdU incorporation and CD34 expression were evaluated using immunofluorescence at 48 hours after injection. The arrowhead indicates BrdU incorporation in a CD34-positive cell. (A, B) Scale bar $=25 \mu \mathrm{m}$. (C, D) Scale bar $=10 \mu \mathrm{m}$.

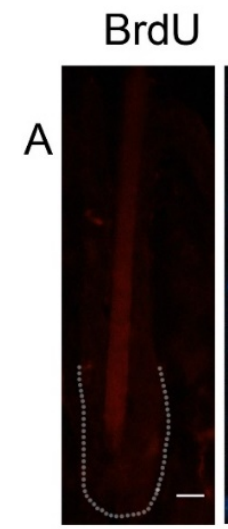

DAPI

Merge

Figure 3. Immunofluorescent detection of BrdU incorporation in the Wntlob-overexpressing hair follicles. Eight-week-old C57BL/6 mice were intradermally injected with AdWnt10b (A, C, E) or AdGFP (B, D, F). BrdU was administered 4 hours before the skin was harvested. BrdU incorporation was determined by immunofluorescence at 12 hours (A, B), 24 hours (C, D) and 48 hours $(E, F)$ after injection. The dotted line outlines the bulge
area. The arrowheads indicate the incorporation of BrdU. Scale bar $=10 \mu \mathrm{m}$.

C

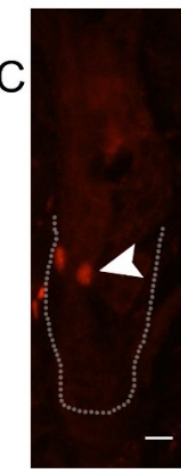

E

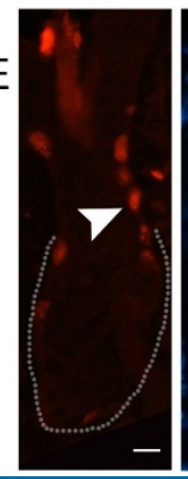

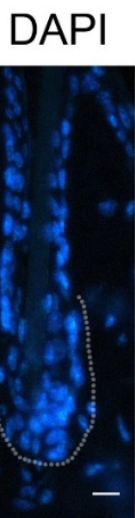
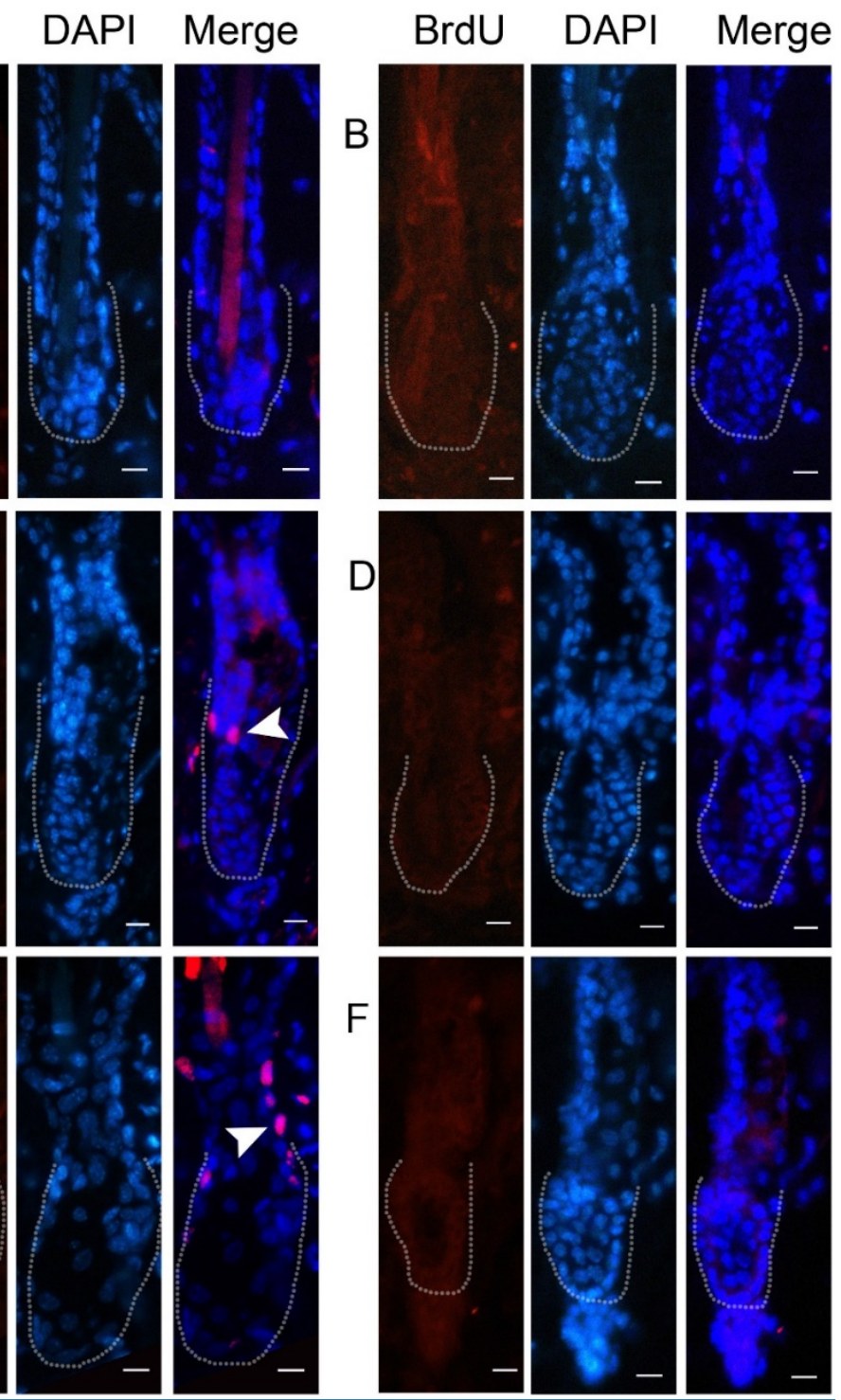

$\mathrm{F}$
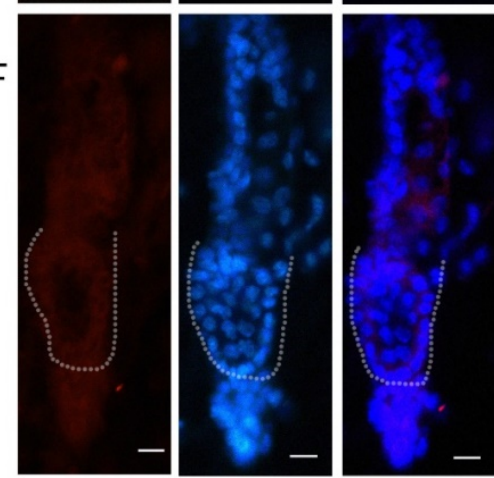
A

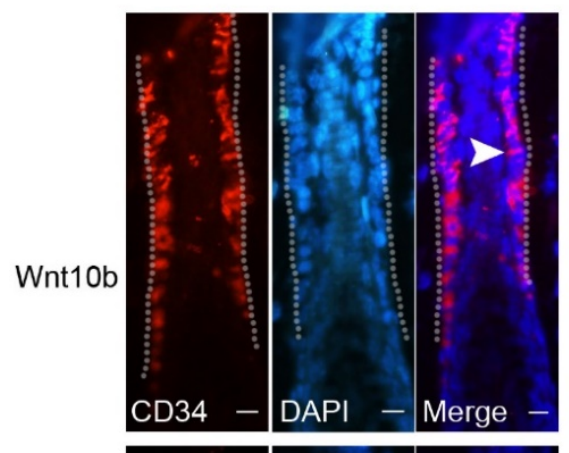

B

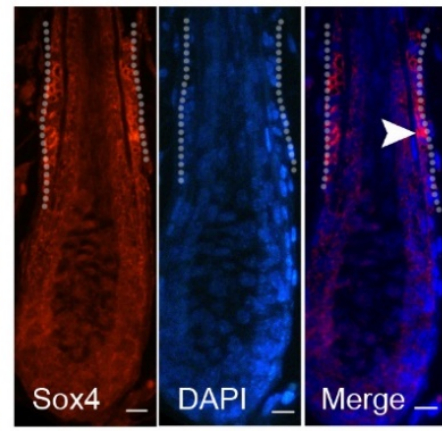

C

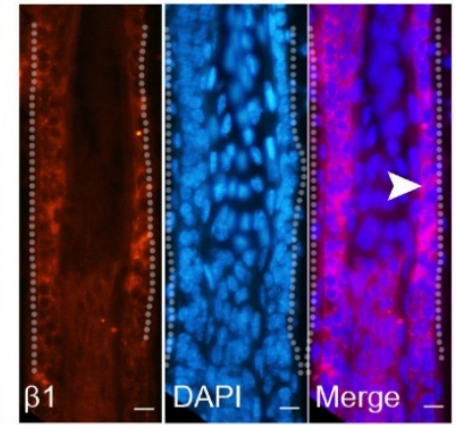

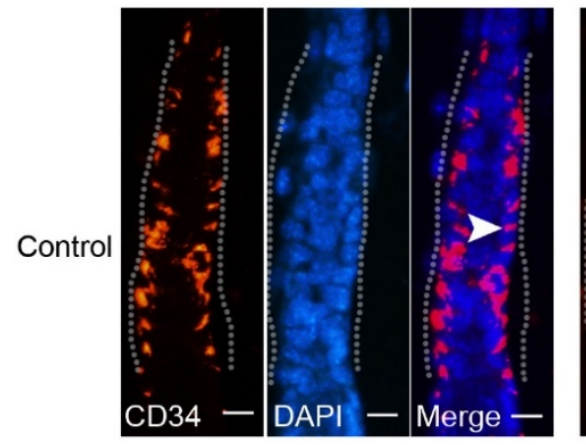
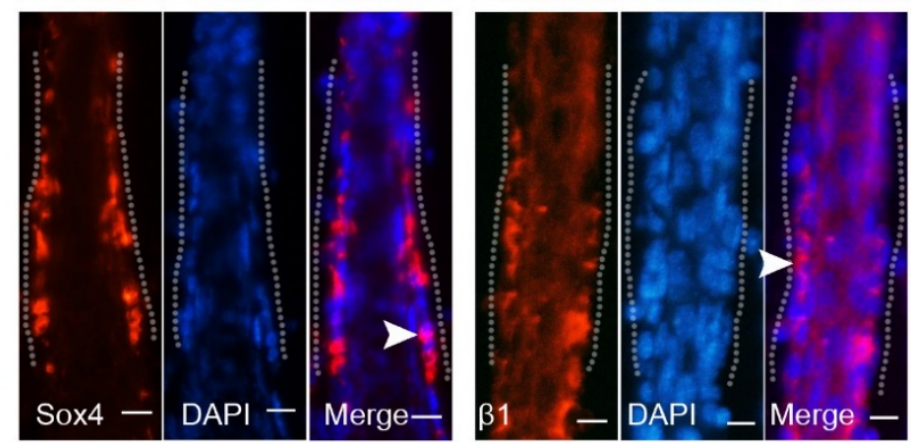

Figure 4. Expression of HFSC markers in Wnt10b-overexpressing hair follicles. Eight-week-old C57BL/6 mice were intradermally injected with AdWnt10b. The expression levels of CD34 (A), Sox4 (B) and B1-integrin (C) were evaluated by immunofluorescence at 7 days after injection. The dotted line outlines the bulge area. The arrowheads representatively indicate positive expression of these markers. Normal anagen hair follicles at the corresponding stage were used as controls. Scale bar $=10 \mu \mathrm{m}$.

As an analog of thymidine, BrdU is incorporated into the newly synthesized DNA of replicating cells by substituting for thymidine during DNA replication. The expression of BrdU thus identifies cells that are actively replicating their DNA [15]. We administered BrdU 4 hours before harvesting the skin of mice; thus, the expression of BrdU in these samples revealed cells that had replicated in those 4 hours. Although the bulge area includes several cell types, because nearly all of the cells are HFSCs, cells in this area are generally considered HFSCs. Here, we found that HFSCs comprised the first round of proliferating cells after the overexpression of Wnt10b. Other studies have also reported that Wnt receptors are present on HFSCs [16]. These findings suggest that HFSCs are among the target cells of overexpressed Wnt10b.

Typically, HFSCs differentiate into hair follicle cells, but under special conditions, they also differentiate into sebaceous glands and epidermal cells $[17,18]$. HFSCs can even be used to aid wound healing in the skin [19]. At a later stage, proliferating cells can be observed in both the distal and proximal parts of the bulge area of Wnt10b-induced hair follicles. This finding implies that overexpressed Wnt10b not only promotes the proliferation of HFSCs but also induces the differentiation of HFSCs into hair follicles and epidermis. This possibility should be tested in future studies.

Adenovirus vectors are commonly used as transient expression vectors for gene editing [20]. After injection, Wnt10b expression is upregulated, peaks at 48 hours after injection, and then decreases gradually. The exogenous expression of Wnt $10 \mathrm{~b}$ continues at a low level for about one month [10]. When we detected the expression of Wnt10b in full anagen 7 days after injection, the expression pattern of Wnt10b in Wnt10b-induced hair follicles was similar to that in cells in the corresponding hair follicle stage. This phenomenon might have resulted from the strong expression of endogenous Wnt $10 \mathrm{~b}$ at that stage. However, the expression patterns of $\beta$-catenin were different. The translocation of $\beta$-catenin from the cytoplasm to the nucleus could still be observed in the hair precortex cells of Wnt10b-induced hair follicles. This observation indicates that the overexpressed Wnt10b was still activating the canonical Wnt signaling pathway and thus promoting the growth of hair follicles. Previously, we reported that Wnt10b-induced hair follicles are larger than normal hair follicles of the corresponding stage, but we were unable to provide a reason at that time [10]. It has since been reported that larger hair follicles induced by prolonged overexpression of $W n t 10 \mathrm{~b}$ resulted from the continuous activation of the $\mathrm{Wnt} / \beta$-catenin signaling pathway [21]. The current results suggest that the larger hair follicles induced by transient overexpression of Wnt10b may also result from activation of $\beta$-catenin. 

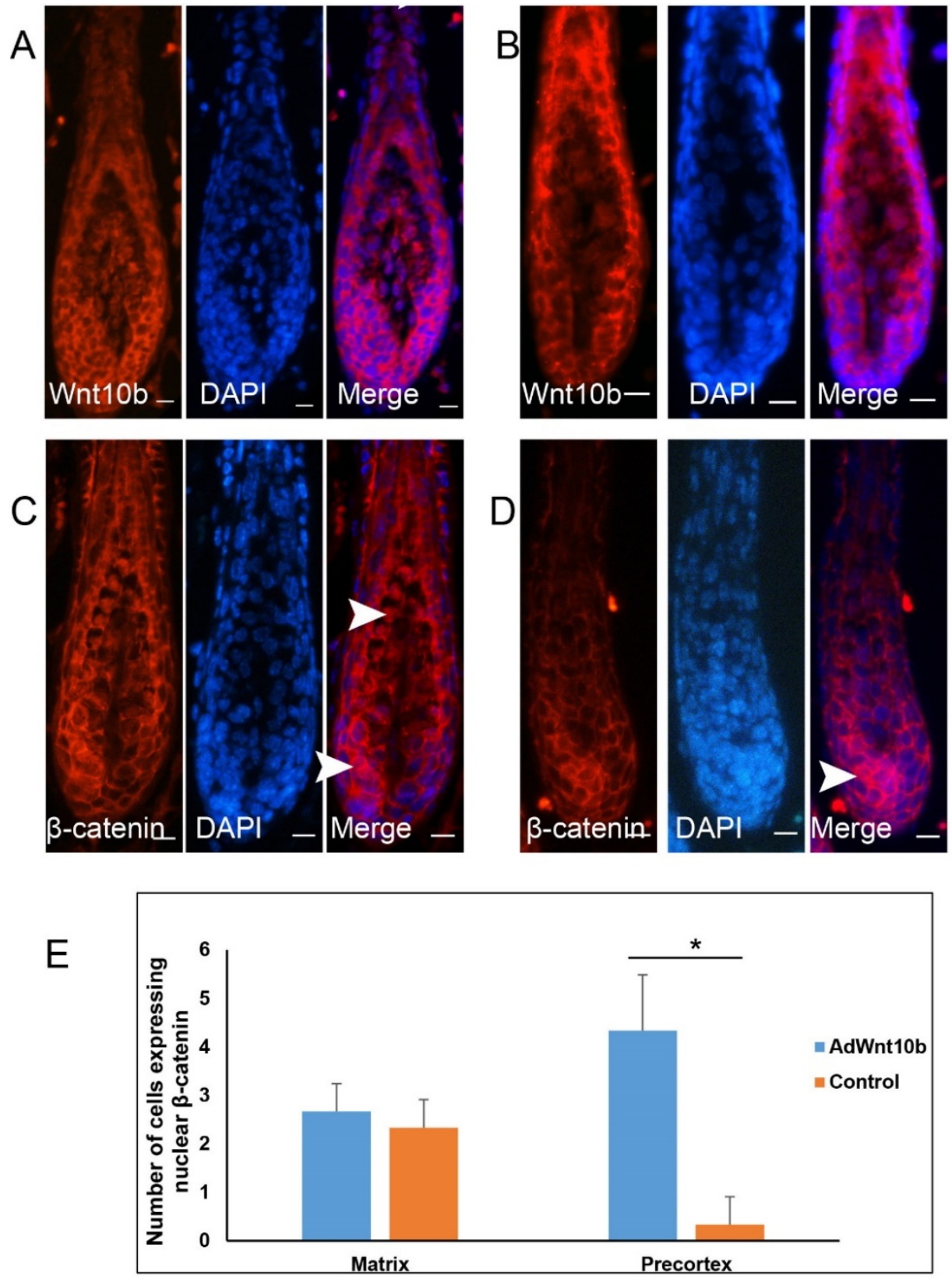

Figure 5. Expression patterns of Wnt10b and $\beta$-catenin in Wnt10b-induced anagen hair follicles. Eight-week-old C57BL/6 mice were intradermally injected with AdWnt10b. Wnt lob $(A)$ and $\beta$-catenin $(C)$ expression levels were determined by immunofluorescence at 7 days after injection. Normal anagen hair follicles at the corresponding stage were used as controls (B, D). The arrowheads indicate the nuclear expression of $\beta$-catenin. Scale bar $=10 \mu \mathrm{m}$. $(\mathrm{E}) \mathrm{Quantitative}$ analysis of $(\mathrm{C})$ and $(\mathrm{D})$. $N=3$, *P< 0.05 .

Because prolonged overexpression of Wnt10b may result in abnormal hair [21], we analyzed the expression patterns of HFSC markers in hair follicles with transiently induced Wnt10b overexpression. The expression patterns of CD34 and $\beta 1$-integrin were similar to those in the control hair follicles of the corresponding stage [22-24]. The different expression levels of Sox4 may have resulted from different sub-stages of anagen, and the control group was at a later sub-stage of anagen. This finding implies that the HFSCs in our model were normal.

In summary, we demonstrated that the overexpression of Wnt10b might activate HFSCs and thus induce hair follicle regeneration. We also demonstrated that Wnt10b might continuously activate the canonical Wnt signaling pathway and thus lead to the larger sizes of Wnt10b-induced hair follicles. This work may help uncover the mechanisms of stem cell activation and aid research related to therapies for diseases associated with dysfunctions of hair follicle regeneration.

\section{Abbreviations}

AdWnt10b: mouse Wnt10b cDNA mediated by adenovirus; AdGFP: green fluorescence protein mediated by adenovirus. 


\section{Acknowledgments}

This work was supported by the National Natural Science Foundation of China (No. 81472895) and the Municipal Natural Science Foundation of Chongqing (No. cstc2013jcyjA10084). We thank Dr. Tong-Chuan $\mathrm{He}$ at Chicago University for the adenovirus production and technical assistance. We thank Prof. Cheng-Ming Chuong at the University of Southern California for advice about this project.

\section{Competing Interests}

The authors have declared that no competing interest exists.

\section{References}

1. Alonso L and Fuchs E. The hair cycle. J Cell Sci. 2006; 119(Pt 3): 391-3.

2. Greco V, Chen T, Rendl M, et al. A two-step mechanism for stem cell activation during hair regeneration. Cell Stem Cell. 2009; 4(2): 155-69.

3. Garcin CL, Ansell DM, Headon DJ, et al. Hair follicle bulge stem cells appear dispensable for the acute phase of wound re-epithelialisation. Stem Cells. 2016.

4. Lei MX, Chuong CM, and Widelitz RB. Tuning Wnt signals for more or fewer hairs. J Invest Dermatol. 2013; 133(1): 7-9.

5. Plikus MV, Mayer JA, de la Cruz D, et al. Cyclic dermal BMP signalling regulates stem cell activation during hair regeneration. Nature. 2008; 451(7176): 340-4

6. Yue $\mathrm{Z}$, Jiang $\mathrm{TX}, \mathrm{Wu} \mathrm{P}$, et al. Sprouty/FGF signaling regulates the proximal-distal feather morphology and the size of dermal papillae. Dev Biol. 2012; 372(1): 45-54

7. Wang X, Tredget EE, and Wu Y. Dynamic signals for hair follicle development and regeneration. Stem Cells Dev. 2012; 21(1): 7-18.

8. Oshimori $\mathrm{N}$ and Fuchs E. Paracrine TGF-beta signaling counterbalances BMP-mediated repression in hair follicle stem cell activation. Cell Stem Cell. 2012; 10(1): 63-75.

9. Xing YZ, Wang RM, Yang $\mathrm{K}$, et al. Adenovirus-mediated Wnt5a expression inhibits the telogen-to-anagen transition of hair follicles in mice. Int J Med Sci. 2013; 10(7): 908-14

10. Li YH, Zhang K, Yang K, et al. Adenovirus-mediated Wnt10b overexpression induces hair follicle regeneration. J Invest Dermatol. 2013; 133(1): 42-8.

11. Li YH, Zhang K, Ye JX, et al. Wnt10b promotes growth of hair follicles via a canonical Wnt signalling pathway. Clinical and Experimental Dermatology. 2011; 36(5): 534-540.

12. Ouji $Y$, Ishizaka S, and Yoshikawa M. Dermal papilla cells serially cultured with Wnt-10b sustain their hair follicle induction activity after transplantation into nude mice. Cell Transplant. 2012; 21(10): 2313-24.

13. Guo $H$, Xing $Y$, Liu $Y$, et al. Wnt/ $\beta$-catenin signaling pathway activates melanocyte stem cells in vitro and in vivo. J Dermatol Sci. 2016; 83(1): 45-51

14. Lei M, Lai X, Bai X, et al. Prolonged overexpression of Wnt10b induces epidermal keratinocyte transformation through activating EGF pathway. Histochem Cell Biol. 2015; 144(3): 209-21.

15. Lauand C, Niero EL, Dias VM, et al. Cell cycle synchronization and BrdU incorporation as a tool to study the possible selective elimination of ErbB1 gene in the micronuclei in A549 cells. Braz J Med Biol Res. 2015; 48(5): 382-91.

16. Lien $\mathrm{WH}$, Polak $\mathrm{L}$, Lin $\mathrm{M}$, et al. In vivo transcriptional governance of hair follicle stem cells by canonical Wnt regulators. Nat Cell Biol. 2014; 16(2): 179-90.

17. Tiede S, Kloepper JE, Bodo E, et al. Hair follicle stem cells: walking the maze. Eur J Cell Biol. 2007; 86(7): 355-76.

18. Lavker RM, Sun TT, Oshima $\mathrm{H}$, et al. Hair follicle stem cells. J Investig Dermatol Symp Proc. 2003; 8(1): 28-38.

19. Leiros GJ, Kusinsky AG, Drago H, et al. Dermal papilla cells improve the wound healing process and generate hair bud-like structures in grafted skin substitutes using hair follicle stem cells. Stem Cells Transl Med. 2014; 3(10): 1209-19.

20. Yamamoto $\mathrm{H}$, Ishimura $\mathrm{M}$, Ochiai $\mathrm{M}$, et al. BTK gene targeting by homologous recombination using a helper-dependent adenovirus/adeno-associated virus hybrid vector. Gene Ther. 2015.

21. Lei M, Guo H, Qiu W, et al. Modulating hair follicle size with Wnt10b/DKK1 during hair regeneration. Exp Dermatol. 2014; 23(6): 407-13.

22. Poblet E and Jimenez F. CD10 and CD34 in fetal and adult human hair follicles: dynamic changes in their immunohistochemical expression during embryogenesis and hair cycling. Br J Dermatol. 2008; 159(3): 646-52.

23. Kadaja M, Keyes BE, Lin M, et al. SOX9: a stem cell transcriptional regulator of secreted niche signaling factors. Genes Dev. 2014; 28(4): 328-41.
24. DeRouen $\mathrm{MC}$, Zhen $\mathrm{H}$, Tan $\mathrm{SH}$, et al. Laminin- 511 and integrin beta- 1 in hair follicle development and basal cell carcinoma formation. BMC Dev Biol. 2010; 10:112. 